The University of Maine

\title{
DigitalCommons@UMaine
}

Spatial Informatics Faculty Scholarship

Spatial Informatics

2013

\section{Indoor Inertial Waypoint Navigation for the Blind}

Timothy H. Riehle

Shane M.Anderson

Patrick A. Lichter

William E. Whalen

Nicholas A. Giudice

University of Maine - Main, nicholas.giudice@maine.edu

Follow this and additional works at: https://digitalcommons.library.umaine.edu/sie_facpub

Part of the Cognition and Perception Commons, and the Graphics and Human Computer Interfaces Commons

\section{Repository Citation}

Riehle, Timothy H.; Anderson, Shane M.; Lichter, Patrick A.; Whalen, William E.; and Giudice, Nicholas A., "Indoor Inertial Waypoint Navigation for the Blind" (2013). Spatial Informatics Faculty Scholarship. 7.

https://digitalcommons.library.umaine.edu/sie_facpub/7 


\title{
Indoor Inertial Waypoint Navigation for the Blind
}

\author{
Timothy H. Riehle, Member, IEEE, Shane M. Anderson, Member, IEEE, \\ Patrick A. Lichter, Member, IEEE, William E. Whalen, Nicholas A. Giudice
}

\begin{abstract}
Indoor navigation technology is needed to support seamless mobility for the visually impaired. This paper describes the construction and evaluation of an inertial dead reckoning navigation system that provides real-time auditory guidance along mapped routes. Inertial dead reckoning is a navigation technique coupling step counting together with heading estimation to compute changes in position at each step. The research described here outlines the development and evaluation of a novel navigation system that utilizes information from the mapped route to limit the problematic error accumulation inherent in traditional dead reckoning approaches. The prototype system consists of a wireless inertial sensor unit, placed at the users' hip, which streams readings to a smartphone processing a navigation algorithm. Pilot human trials were conducted assessing system efficacy by studying route-following performance with blind and sighted subjects using the navigation system with real-time guidance, versus offline verbal directions.
\end{abstract}

\section{INTRODUCTION}

A wide assortment of technologies has been proposed to develop indoor navigation systems for the blind and vision impaired. Proximity-based and triangulation systems have been successfully demonstrated [1] and employed. Despite the technical success of these technologies, broad adoption has been limited due to their significant infrastructure and maintenance costs (for review, see Giudice and Legge, 2008 [2]). The approach explored in this research seeks to solve this infrastructure cost problem by utilizing only body-worn inertial sensors, MEMS accelerometers and gyros, combined with a smartphone processing a navigation algorithm. The approach described here is one component of an ongoing project that will ultimately fuse several complementary indoor navigation technologies together. In recent publications, the authors have described and demonstrated another component of this project utilizing magnetic sensing of indoor magnetic anomalies to localize the traveler along an indoor route [3][4]. To optimize each navigation component, a complete indoor navigation solution has been constructed and tested using only the current technology under development. This approach has provided our team with frequent opportunities to demonstrate our technology and user interface with potential users.

Manuscript received February 4, 2013. This work was supported in part by the National Eye Institute under grant 2R44EY017228-02A2.

T. Riehle is with Koronis Biomedical Technologies, Inc. Maple Grove, MN 55369, USA (phone: 888-274-1317; fax: 763-463-9000; e-mail: triehle@koronisbiotech.com)

S. Anderson and P. Lichter are with Koronis Biomedical Technologies, Inc. Maple Grove, MN, 55369, USA

$\mathrm{W}$. Whalen is a doctoral student at the University of Maine

N. Giudice is Associate Professor, School of Computing and Information Science, University of Maine, Orono, ME 04469-5711, USA (e-mail: Nicholas.giudice@maine.edu)
Indoor navigation technology is needed to support seamless mobility for the visually impaired. Most people who are blind or have low vision can navigate outdoors using a cane, guide dog or their own low vision as an aid, but indoor navigation in large or unfamiliar buildings can be very challenging. To be done accurately, it requires reading signs, room numbers, building maps, and/or identifying landmarks, tasks which are difficult or impossible for a person with low vision. A significant problem in indoor navigation for the visually impaired relates to orientation information: knowing current position in the building and updating changing position/heading with movement. The biggest challenge to low-vision navigation is not mobility information, awareness and avoidance of obstructions to the path of travel, or in executing routes, but with spatial updating, spatial inference and cognitive map development [5].

\section{II.PEDESTRIAN DEAD RECKONING APPROACH}

The navigator technology described here is based on a pedestrian dead reckoning (PDR) approach. PDR algorithms combine step detection with heading estimation to compute changes in position. Body worn accelerometers are sensitive to the characteristic motions involved with walking and this data can be processed to detect steps. Step detection is coupled with an individual's measured gait length to provide an estimate of the distance traveled with each step. Sophisticated PDR approaches include algorithms to estimate the change in gait length as an individual modifies their stride.

Heading is typically measured by magnetometers sensing the Earth's field, or gyroscopes which sense angular velocity. When combined with the accelerometer's measurement of the gravity down vector, the components of the magnetic field or angular velocity in the horizontal plane provide an estimation of heading.

Given a known starting point and a measured gait length, PDR can provide a users' approximate real-time position without relying on external infrastructure. This approach, however, suffers from error accumulation. Small errors in gait length and heading slowly accumulate and eventually overwhelm the position estimate. Dead reckoning is typically paired with localization technologies that do not suffer from error accumulation, such as GPS, to avoid this problem.

The approach developed here uses the trajectory of the planned route to supply additional information to the navigator algorithms, enabling the problem of error accumulation to be controlled. A planned route is composed of straight-line segments and expected heading changes. PDR is used within straight-line segments to update the distance along the segment. Error accumulation is controlled by comparing sensed turns to map features. As the traveler 
executes an expected turn the system detects the heading change and updates the current position estimate to the start of the next route segment. In this way, the PDR position estimate is only used for relatively short segments, typically less than $100 \mathrm{~m}$. This approach is well suited to the modest consumer grade sensors found in smartphones, and can work without foot or shin mounted sensors, common in PDR approaches.

The system's user interface consists of text-to-speech audio cues associated with map features, and triggered based on proximity. As the user traverses a route segment the system announces directional cues such as upcoming turns as well as nearby points of interest. This approach is well suited to the needs of blind travelers who may have excellent mobility skills, and are sensitive to subtle environmental cues such as changes in acoustics. Announcing nearby features can both reinforce cognitive map development as well as facilitate innate orientation skills to increase overall navigation accuracy.

\section{EXPERIMENTAL METHODS}

\section{A. Sensor Hardware}

The hardware utilized in the pilot human trials consisted of custom wireless inertial measurement units (IMUs) and Android smartphones. Figure 1 shows the Bluetooth IMU that was used in this research. Acceleration was sensed using a LIS352AX 3-axis MEMS accelerometer and angular rotation was

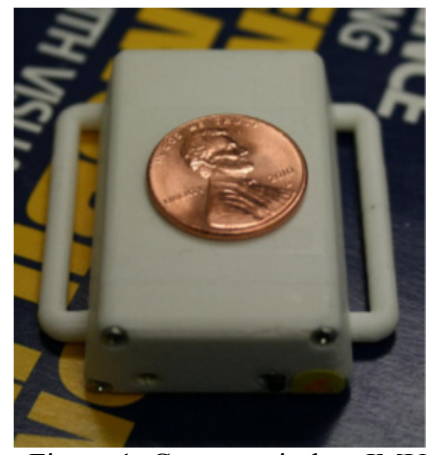

Figure 1: Custom wireless IMU used to instrument subjects in this research. sensed using a LPR430AL 2-axis and a LY330ALH 1-axis gyroscope, all manufactured by ST Microelectronics. Sensor data was sampled and transmitted at $200 \mathrm{~Hz}$ to a smartphone. Figure 3 shows the final navigator application operating on a Google Nexus 4 smartphone. The use of the wireless IMU was opted for instead of the smartphone's integrated accelerometer and gyroscope to ensure greater control over the placement and orientation of the sensors during testing. IMUs were worn on the subjects' hip for the tests described below. In principle, however, the methods described are suitable for, and were developed to use the integrated sensors on modern smartphones.

\section{B. Map Construction}

The focus for the research described here is the tracking of individuals as they traverse routes designed to take them between fixed starting and ending positions within a building, based on mapped routes. Map creation started with design of four approximately equally complex routes (to facilitate counterbalancing in the pilot human trials.) Next, the routes were surveyed using an inexpensive measuring wheel to measure the length of straight-line segments, and the position of points of interest within these segments. The change of heading between segments was estimated without aid of instruments, and refined using Google Maps of the Mall. Figure 2 shows a simple route surveyed at the Mall of

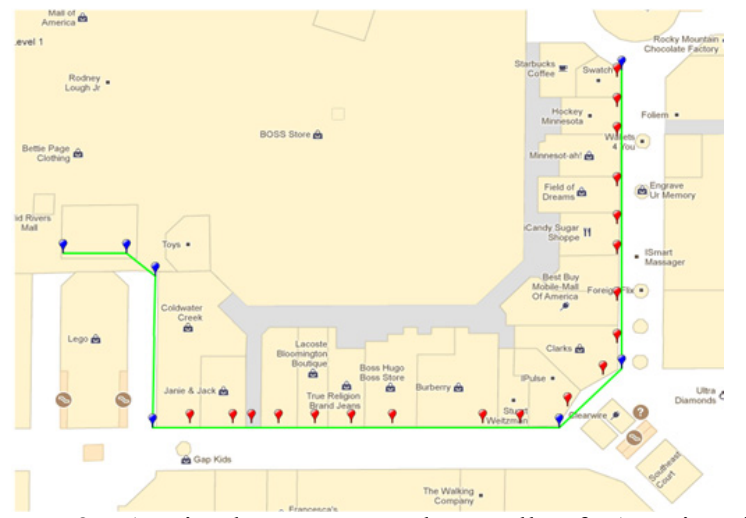

Figure 2: A simple route at the Mall of America in Bloomington, MN, overlaid on the Google Map for the Mall. The blue markers outline the straight-line segments; the red markers show the location of the points of interest.

America. The route follows the edge of the hallway, reflecting the mobility technique called shorelining used by white cane users.

\section{Step Detection}

An approach to step detection was developed that recognized the practical limitations associated with the application goal of utilizing smartphone sensors. Common approaches that seek to optimize sensitivity to step detection and stride length estimation commonly choose the foot or the shin for sensor placement. Lower leg placement is sensitive to the legs swing angle as well as the different phases of the stride. This placement is not feasible for a smartphone application, so instead the upper body placement of the sensor was studied. One important difference between lower leg and upper body placement is that upper body placement detects the motion from both left and right steps, more or less equally, and the acceleration signal has fewer high frequency components associated with the foot plant.

Using a dataset of 3-axis accelerometer data collected at the hip during normal walking activities, indoor and outdoor, and with varying speeds, a number of candidate step detection algorithms were implemented and tested. Libby's method [6], a PanTompkins algorithm [7], and Wolf's Method [8] were compared to a simple threshold based approach. The simple threshold approach analyzes the magnitude of the low-pass filtered acceleration vector, and requires a positive excursion above $1 G$ plus threshold following a negative excursion of $1 G$ minus threshold. The use of acceleration magnitude has the benefit of rotational invariance, avoiding the need to place the sensor in some preferred orientation. For ordinary walking

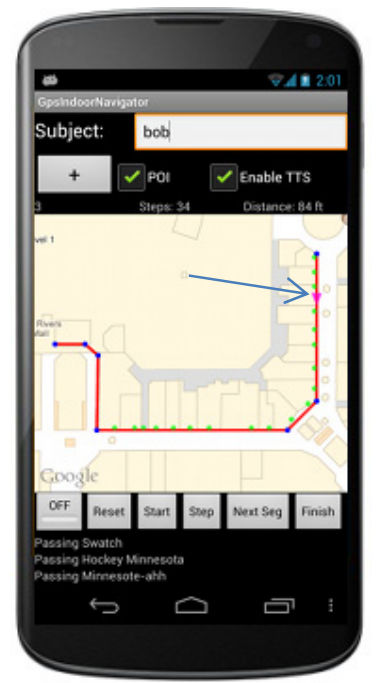

Figure 3: Navigator application running on a Nexus 4 smartphone and showing users current position estimate. 


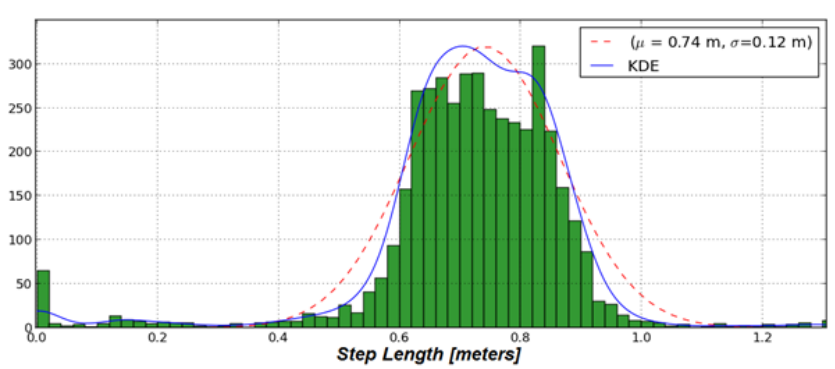

Figure 4: Measured step lengths from the training dataset overlaid with a simple Gaussian fit, and a kernel density estimate.

motion the simple threshold algorithm performed comparably to the more sophisticated methods. Due to its simplicity and adequate performance, the simple threshold algorithm was selected for use in the final navigator application.

Once an algorithm for step detection had been selected, a study was performed to determine if a hip mounted sensor could infer changes in gait length. Two datasets were collected for this study using a single subject. An outdoor dataset with normal walking was collected using a centimeter-scale accuracy differential GPS system to provide a direct measurement of stride length. A second dataset was gathered using a treadmill set at fixed speeds, allowing the stride length to be estimated using the time between steps inferred from the step detection algorithm. Figure 4 shows the distribution of step lengths for the test subject, and shows the step length variance.

To develop a step length estimator 52 variables were measured during each step, such as step duration, sensor variances, maximum sensor values, derivatives of the acceleration, together with changes in these quantities between steps. The correlation between these variables and the measured step length was performed to identify the variables with the greatest sensitivity. Figure 5 shows the four variables with the highest correlation together with the data scatterplots. Step length estimation was not used for the final navigator pilot human evaluations described below, and is still under development.

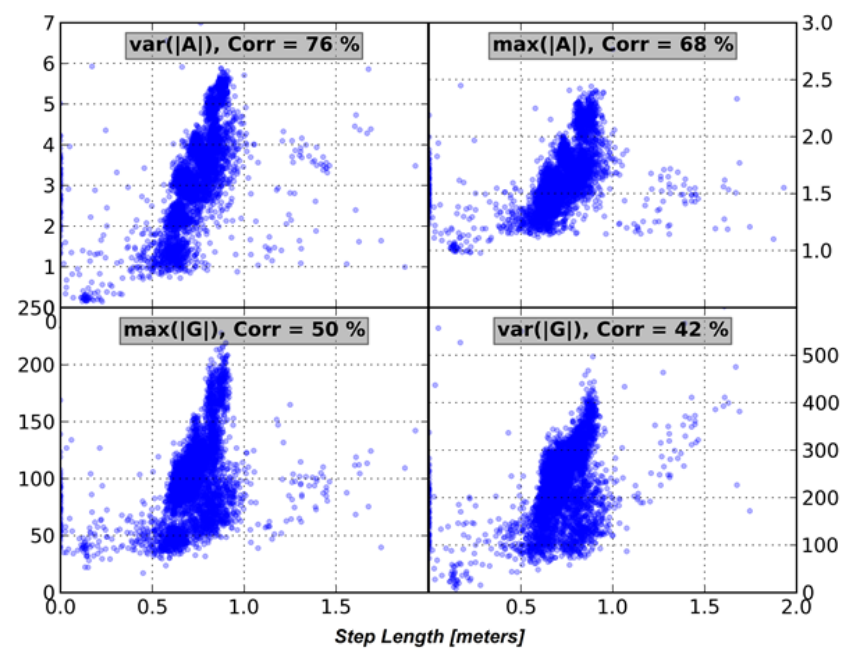

Figure 5: Scatter plots of the four variables with highest correlation to step length: (upper left) accelerometer variance per step, (upper right) maximum acceleration (in G's) per step, (lower left) maximum angular velocity per step (lower right) variance of angular velocity per step.

\section{Heading Estimation}

Heading was inferred using the approach developed by Madgwick, et al. [9]. This approach uses a quaternion representation for orientation and is computationally efficient and relatively easy to implement. Both the Magnetic Angular Rate and Gravity (MARG) and the gyroscope-only IMU approaches were implemented and tested in both indoor and outdoor environments. The MARG is a complementary filter that mixes the orientation estimate obtained by integrating the gyroscope measurements together with the estimate from the accelerometer and magnetometer. In contrast, the IMU filter does not use a magnetometer and only provides a relative orientation measurement; unlike MARG it must be given a starting heading estimate.

In outdoor environments both implementations performed acceptably, although the IMU filter showed a small drift over time, about 10 degrees per minute. In indoor environments with strong magnetic anomalies the MARG orientation estimate showed unacceptable heading accuracy. It was concluded that the IMU filter, despite its potential for drift, provided the best orientation solution for indoor environments due to its immunity to magnetic distortions.

\section{WAYPOINT NAVIGATION ALgORITHMS}

The goal of the waypoint navigator is to compute position estimates based on the heading, step detection and measured step lengths. Knowledge of the users planned route trajectory offers additional information to control the error accumulation inherent in dead reckoning techniques.

Route maps, such as that shown in Figure 2, are represented by straight line segments of a given length and heading, and followed by a known heading change to the next segment. While a user is traveling on a segment the navigational problem is considered to be one dimensional: step counting was used to estimate distance along the segment. As points of interest are reached the navigator's user interface was triggered to announce these features via text-to-speech. As the end of a segment was neared, the upcoming turn was announced ahead of the turn to both allow the user to use their own skills to detect the turn, as well as to accommodate potential position errors due to error accumulation.

Turn detection was used to ensure the user stays on course. While the user was walking along a straight line segment heading estimates from the IMU filter, averaged per step are used to form both an estimate of the recent heading from the last $\mathrm{N}$ steps, as well as a long term heading average from the previous steps along the segment, excluding the most recent $\mathrm{N}$ steps. The difference between the short term and long term heading is used to compute the turn estimator $T_{i}$ at step $i$, shown in Eq. 1. This formulation has the advantage of being insensitive to long term heading drift, which is expected from the IMU filter.

$$
T_{i}=\sum_{j=i-N}^{i}\langle H\rangle_{j}-\sum_{\text {step } j=0}^{i-N-1}\langle H\rangle_{j}
$$

If a heading change greater than a minimum threshold is detected while on a segment then an off-route indication is triggered. As the user approaches the end of a segment the heading change associated with the expected turn is looked 
for. If a change in course is detected that is within tolerance of the expected turn then the navigator uses that information to update the users' position at the start of the next segment, thus correcting for any accumulated errors. Finally, if the expected turn is not sensed an off-route indication is triggered if the position estimate overshoots the segment length by some tolerance. Step lengths were not adjusted, based on measured over or undershoot, due to the concern that such a feature would require extensive testing for minimal increase in performance.

\section{Pilot Human Study}

The feasibility study for the prototype navigator was assessed in a pilot human behavioral experiment carried out in the Mall of America, in Bloomington, MN (see Figure 2.) The goal of this feasibility study was to demonstrate the efficacy of our approach for supporting real-time guidance in a challenging environment where end-users actually want to travel. Eight blind individuals who were either totally blind or only had very limited light perception participated in the study. All self-reported as being highly independent travelers. In addition, eight sighted individuals participated as controls in the study, for a total of $\mathrm{N}=16$ subjects, ranging in age from 18 to 59 years.

After a practice session where participants were familiarized with the experimental apparatus and task, including calibration of the system to the participant's stride length and a test run with the system with corrective feedback, they began the experimental trials. During the route navigation phase, participants were started at one of the four pre-determined route origin locations in the Mall and asked to find a route to an unknown destination target location (a specific store entrance). Route navigation occurred in two conditions (condition by route order was counter-balanced between participants). In the "System Aided" condition, participants walked along the route with real-time assistance from the system describing what stores they were passing, alerting them to salient landmarks along the route, the length of each route segment (in feet), route deviations (decision points), and describing the actions to perform at these decision points. In the "Unaided Memory" condition, participants received the same verbal instructions (minus the store names which imposed an undue cognitive load) but rather than hearing this information sequentially in real-time as they walked the route, the instructions were provided all at once at the route's origin. For both conditions, the experimenter served as a bystander who could provide critical information if the participant got disoriented or felt they needed additional assistance, similar to what might be requested from a random passerby during independent travel (see [4] for more detail on our methodology.)

\section{A. Results}

The results provide compelling support for the efficacy of using the system to navigate through our highly complex experimental setting. Where six out of eight participants in the unaided memory condition made bystander requests, for a total of 26 requests, only 4 total requests were made in the system aided condition. Comparing the temporal duration required to navigate the routes between conditions also yielded marked differences. Where blind participants in the system aided condition took an average of $221 \mathrm{~s}$ to traverse the routes, the same routes took $300 \mathrm{~s}$ in the unaided memory condition. A paired sample T-test confirmed that these differences between conditions were statistically reliable, $t(15)=-2.64$, two-tail, $p=0.018$. In addition, six out of eight trials in the system aided condition led to correct localization of the route's destination (with the two misses yielding small localization errors on the order of feet based on calibration noise). By contrast, only two of eight trials in the unaided memory condition yielded correct localization.

Comparing performance between the blind and sighted participants was also informative. The sighted participants took an average of $213 \mathrm{~s}$ to traverse the routes. Independent sample T-tests revealed that their performance reliably differed from the blind participants in the unaided memory condition, $\mathrm{t}(14)=6.48$, two-tail, $\mathrm{p}=0.016$ but that the sighted performance was insignificant compared to the blind participants in the system aided condition, $\mathrm{t}(14)=8.12$, twotail, $p=0.637$. These results provide clear empirical evidence that blind travelers using real-time route information from our system can perform on-par with their sighted counterparts. The convergence of statistical performance measures and enthusiasm from the subjects after using the system, combined with the unique advantages of inertial navigation technology demonstrates the importance of providing real-time guidance information and suggests that our system is a promising approach for future development.

\section{ACKNOWLEDGMENT}

The authors would like to express their thanks to the owners and operators of the Mall of America for allowing use of their site for data collection and human testing.

\section{REFERENCES}

[1] T. H. Riehle, P. Lichter, and N. A. Giudice, "An indoor navigation system to support the visually impaired," in the $30^{\text {th }}$ Annual Engineering in Medicine and Biology Society, 2008. , pp. 4435-4438.

[2] N. A. Giudice and G. E. Legge, "Blind navigation and the role of technology," The Engineering Handbook of Smart Technology for Aging, Disability, and Independence, pp. 479-500, 2008.

[3] T. H. Riehle, S. M. Anderson, P. A. Lichter, J. P. Condon, S. I. Sheikh, and D. S. Hedin, "Indoor waypoint navigation via magnetic anomalies," in the $33^{\text {th }}$ Annual Engineering in Medicine and Biology Society, EMBC, pp. 5315-5318.

[4] T. H. Riehle, S. M. Anderson, P. A. Lichter, N. A. Giudice, S. I. Sheikh, R. J. Knuesel, D. T. Kollmann, and D. S. Hedin, "Indoor magnetic navigation for the blind," in the $34^{\text {th }}$ Annual International Conference of the IEEE Engineering in Medicine and Biology Society (EMBC), 2012, pp. $1972-1975$.

[5] C. Thinus-Blanc and F. Gaunet, "Representation of space in blind persons: Vision as a spatial sense?," Psychological Bulletin, vol. 121, no. 1, p. 20, 1997.

[6] R. Libby, A Simple Method for Reliable Footstep Detection in Embedded Sensor Platforms. 2009

[7] H. Ying, C. Silex, A. Schnitzer, S. Leonhardt, and M. Schiek, "Automatic step detection in the accelerometer signal," in 4th International Workshop on Wearable and Implantable Body Sensor Networks (BSN 2007), 2007, pp. 80-85.

[8] M. Marschollek, M. Goevercin, K. H. Wolf, B. Song, M. Gietzelt, R. Haux, and E. Steinhagen-Thiessen, "A performance comparison of accelerometry-based step detection algorithms on a large, nonlaboratory sample of healthy and mobility-impaired persons," in the $30^{\text {th }}$ Annual Engineering in Medicine and Biology Society, 2008., pp. 1319-1322

[9] S. O. H. Madgwick, A. J. L. Harrison, and R. Vaidyanathan, "Estimation of IMU and MARG orientation using a gradient descent algorithm," in 2011 IEEE International Conference on Rehabilitation Robotics (ICORR), 2011, pp. $1-7$. 